ISSN 1980-5098

\title{
FUNGOS ECTOMICORRÍZICOS NA PRODUÇÃO DE MUDAS DE Eucalyptus grandis W. Hill ex. Maiden EM NEOSSOLO QUARTZARÊNICO
}

\author{
ECTOMYCORRHIZAL ISOLATES IN THE SEEDLING PRODUCTION OF Eucalyptus grandis \\ W. Hill ex. Maiden IN QUARTZARENIC NEOSOLS
}

\begin{abstract}
Eduardo Lorensi de Souza ${ }^{1}$ Zaida Inês Antoniolli ${ }^{2}$ Rafael Goulart Machado ${ }^{3}$ Daniel Eckhardt Pazzini ${ }^{3}$ Sabrina Fátima Dahmer ${ }^{3}$ Marciel Redin ${ }^{4}$ Maiara Figueiredo Ramires ${ }^{5}$
\end{abstract}

\section{RESUMO}

No estado do Rio Grande do Sul, os reflorestamentos de eucalipto encontram-se, geralmente, em solos de baixa fertilidade natural, favorecendo a associação ectomicorrízica. Neste trabalho, avaliou-se o efeito da inoculação dos isolados de fungos ectomicorrízicos UFSC-Pt116, UFSC-Pt188 e UFSC-SA9, sobre plantas de Eucalyptus grandis, em Neossolo Quartzarênico. As avaliações foram realizadas aos 30, 45, 60, 75 e 90 dias após semeadura. Plantas inoculadas com os isolados UFSC-Pt116, UFSC-Pt188 e UFSC-SA9 alcançaram maior altura, diâmetro do caule, massa seca da parte aérea e volume de raízes em relação ao controle. Plantas inoculadas com o isolado de fungo ectomicorrízico UFSC-Pt188 apresentaram maior teor de fósforo e potássio na parte aérea que as plantas não inoculadas. A inoculação com o isolado fúngico ectomicorrízico UFSC-Pt116 promoveu maior altura e massa seca da parte aérea. Para os teores de nitrogênio e potássio, e para massa seca de raízes não foram observadas diferenças significativas entre os tratamentos. A inoculação com os fungos ectomicorrízicos UFSC-Pt116, UFSC-SA9 e UFSC-Pt188 isoladamente, promoveram maior altura, diâmetro de caule, acúmulo de massa seca da parte aérea e volume de raízes das plantas de Eucalyptus grandis.

Palavras-chave: eucalipto; micorrizas; nutrição mineral.

\begin{abstract}
In Rio Grande do Sul state, the eucalyptus planted forests are cultivated in low fertility soils, which contribute to ectomycorrhizal association. This study evaluated the effect of ectomycorrhizal isolates UFSC-Pt116, UFSC-Pt188 and UFSC-SA9 inoculation, individually and in combination, with Eucalyptus grandis in Quartzarenic Neosols. Evaluations were performed at 30, 45, 60, 75 and 90 days after sowing. Plants inoculated with isolates UFSC-Pt116, UFSC-Pt188 and UFSC-SA9 showed highest height, stem diameter, dry mass of shoot and root volume than the control. Plants inoculated with the ectomycorrhizal fungus isolate UFSC-Pt188 showed highest content of phosphorus and potassium in shoots than non-inoculated plants. Inoculation individual ectomycorrhizal isolate UFSC-Pt116 promoted the highest height and shoot dry mass. For nitrogen and potassium, and root biomass have not showed significant differences between treatments. Then inoculation with ectomycorrhizal UFSC-Pt116, UFSC-Pt188 and UFSC-SA9 alone

1 Engenheiro Agrônomo, Dr., Professor Adjunto da Universidade Estadual do Rio Grande do Sul, Rua Cipriano Barata, 47, CEP 98600-000, Três Passos (RS), Brasil. elorensi@yahoo.com.br

2 Bióloga, Dr ${ }^{a}$., Professora Associada da Universidade Federal de Santa Maria, Av. Roraima, 1000, CEP 97105-900, Santa Maria (RS), Brasil. zantoniolli@gmail.com

3 Engenheiro Agrônomo, MSc., Universidade Federal de Santa Maria, Av. Roraima, 1000, CEP 97105-900, Santa Maria (RS), Brasil.rgoulartmachado@gmail.com/ daniel.pazzini@hotmail.com/ sabrina_dahmer@hotmail.com

4 Engenheiro Agrônomo, Dr., Professor Adjunto da Universidade Estadual do Rio Grande do Sul, Rua Cipriano Barata, 47, CEP 98600-000, Três Passos (RS), Brasil. marcielredin@gmail.com

5 Tecnóloga em Agropecuária Integrada, Doutoranda do Programa de Pós-Graduação em Ciência do Solo, Centro de Ciências Rurais, Universidade Federal de Santa Maria, Rua Tereza Cristina, 15, CEP 98600-000, Três Passos (RS), Brasil.
\end{abstract}

Recebido para publicação em 9/08/2013 e aceito em 20/08/2015 
promoted highest height, stem diameter, dry mass of shoot and plant root volume of Eucalyptus grandis. Keywords: eucalyptus; mycorrhiza; mineral nutrition.

\section{INTRODUÇÃO}

A degradação do solo está ocorrendo em muitas partes do mundo (DORNELES, 2009). No planeta, a ONU cita que existem 2 bilhões de hectares de solos degradados e, desses, 200 milhões estão no Brasil, sofrendo ações como cultivo de solos com aptidão agrícola inadequada, mineração, construção represas, estradas e áreas industriais (EMBRAPA, 2002). A diminuição da produtividade agrícola, migração, insegurança alimentar, prejuízos a recursos e ecossistemas básicos e a perda de biodiversidade genética e de espécies são algumas das consequências que podem vir a ocorrer com os processos de degradação do solo.

Na década de 50, iniciou-se o processo de modernização do campo, principalmente nas regiões Sul e Sudeste, expandindo-se para outras regiões, sobretudo, a partir da década de 70 (TEIXEIRA, 2005). No Rio Grande do Sul (RS) essa modernização trouxe consigo graves consequências em termos de degradação ambiental, tendo na introdução do cultivo da soja e no pisoteio do gado, os principais responsáveis pela intensificação dos processos erosivos do solo (SUERTEGARAY et al., 2001). Quando a vegetação é destruída e a camada de solo fértil é perdida, os processos erosivos avançam e podem trazer resultados como a perda da capacidade produtiva dos solos (DORNELES, 2009). O mesmo autor cita que esse fato é possível de ser observado na região sudoeste do RS, com a problemática dos chamados "desertos", nome pelo qual popularmente se denominam focos de arenização.

A região sudoeste do RS vivencia um processo que vem ocorrendo ao longo dos anos devido à fragilidade natural do ecossistema, que associada à erosão eólica e intensificada pelas práticas inadequadas de manejo do solo, possibilita a formação dos areais. A partir de uma topografia favorável ocorre a formação de ravinas e voçorocas e assim, pela erosão sucede-se a origem dos areais (SUERTEGARAY et al., 2001). Além da fragilidade natural do material geológico e da atuação dos agentes erosivos, a ação antrópica interfere significativamente na degradação do solo (DORNELES, 2009).

$\mathrm{Na}$ região sudoeste do RS existem áreas de Neossolos Quartzarênicos que estão sujeitas aos processos de arenização. Estas áreas são depósitos areníticos inconsolidados, retrabalhados sob os processos característicos do clima. Esta ocorrência está associada ao substrato arenítico, mapeado na região Sudoeste como formação Botucatu, com cobertura vegetal predominante de gramíneas. Moller et al. (1975) afirmaram haver no município de São Francisco de Assis cerca de 432 ha de áreas arenosas. Atualmente, avalia-se que a área foi expandida para mais de 5.000 ha cobertos por dunas, abrangendo dez municípios gaúchos, incluindo, São Francisco de Assis (MELLO, 2006). Segundo este mesmo autor, a fragilidade deste solo é resultado da sua dificuldade em compensar as perturbações impostas pela ação antrópica, notado pela suscetibilidade natural à erosão hídrica e eólica, que contribui para sua degradação.

A espécie florestal Eucalyptus grandis é uma das mais cultivadas no estado do RS, sendo responsável por grande parte do sucesso da atividade florestal brasileira, principalmente nos setores ligados à produção de papel e celulose (EMBRAPA, 2003). O Eucalyptus é o gênero florestal mais cultivado no Brasil possuindo a segunda maior área plantada do mundo, superando 4,7 milhões de ha implantados (SOUZA et al., 2008). O RS representa a $5^{\mathrm{a}}$ maior área totalizando 273.042 ha (ASSOCIAÇÃO BRASILEIRA DOS PRODUTORES DE FLORESTAS PLANTADAS, 2011). As espécies do gênero se caracterizam pela alta produtividade e pela adaptabilidade às mais diversas regiões e climas. Apresentam também potencial para captura de dióxido de carbono $\left(\mathrm{CO}_{2}\right)$ na atmosfera, mitigando o efeito estufa (GARAY et al., 2004).

Com a expansão das áreas destinadas ao florestamento, aumentaram também os sintomas da deficiência de macro e micronutrientes (RODRIGUES et al., 2010). Neste contexto, destacam-se os fungos ectomicorrízicos (fECM) e arbusculares (fMA), que têm a característica de associar-se a plantas como o eucalipto e, que ao associarem-se com essas plantas, desenvolvem no solo, estruturas eficientes para a absorção de água e nutrientes, os quais são posteriormente transferidos às plantas, favorecendo sua sobrevivência e crescimento (MARX, 1969; FERREIRA, 2007). Dentre esses organismos ectomicorrízicos, as ectomicorrizas promovem um incremento significativo da área de absorção radicular das plantas, maximizando o aproveitamento de água e nutrientes, como o fósforo $(\mathrm{P})$, o nitrogênio $(\mathrm{N})$ e o potássio 
(K), bem como alguns micronutrientes não fungistáticos (GLOWA et al., 2003). Com isto, contribuem para o estabelecimento e desenvolvimento das plantas, mesmo em solos com baixa fertilidade ou degradados (SANTOS et al., 2008).

Apesar de ser uma espécie produtiva, os reflorestamentos com eucalipto têm se concentrado em regiões brasileiras de solos com baixa fertilidade (LEITE et al., 2011), especialmente em P. A associação de fECM com as plantas de eucalipto favorece o estabelecimento e a sobrevivência da planta no campo, pelo aumento na capacidade de absorção de nutrientes, aumento da longevidade de raízes, proteção contra patógenos, aumento no rendimento de massa seca e na absorção de P (BARROS et al., 1978). Os benefícios da interação organismo-planta são vantajosos do ponto de vista principalmente no que diz respeito ao crescimento melhor no viveiro e ao estabelecimento inicial de plantas quando transplantadas para regiões desfavoráveis como as áreas em arenização no sudoeste do RS. Além disso, plantas inoculadas com fECM teriam vantagens sobre as não inoculadas no que diz respeito à captura de nutrientes, especialmente de $\mathrm{P}$, que é pouco móvel no solo, facilitando assim o estabelecimento a campo das mudas de eucalipto, em especial em regiões arenizadas.

Considerando a baixa fertilidade destes solos onde as florestas de eucalipto estão sendo implantadas, faz-se a adoção de alternativas econômicas e ambientalmente viáveis, para a produção de plantas sadias destinadas ao florestamento de áreas degradadas. Uma alternativa para produção de mudas de eucalipto é o uso de fECM para posterior transplante para as áreas em processo de arenização, visando aumentar a absorção de nutrientes, promover resistência ao ataque de patógenos e aumentar o índice de sobrevivência destas plantas em solos de baixa fertilidade.

A associação da planta com o fECM aumenta a absorção de nutrientes e água, além de induzir resistência à baixa disponibilidade de água e patógenos (SOUZA et al., 2008). A inoculação com um único isolado fúngico não representa fielmente o que acontece em condições naturais, nas quais se encontram várias espécies de fECM e outros organismos em interação constante. Assim, é importante conhecer esta dinâmica, uma vez que a interação entre diferentes fECM pode ser ainda mais benéfica à produção de plantas de eucalipto. O presente estudo teve por objetivo avaliar o efeito da inoculação com isolados de fECM, individualmente ou em mistura, sobre variáveis de desenvolvimento e muda de Eucalyptus grandis cultivadas em Neossolo Quartzarênico.

\section{MATERIAL E MÉTODOS}

\section{Delineamento experimental}

O delineamento experimental utilizado foi o inteiramente casualizado, com oito tratamentos: T1 - Controle (não inoculado), T2 - UFSC-Pt116, T3 - UFSC-SA9, T4 - UFSC-Pt188, T5 - UFSC-Pt116 + UFSC-SA9, T6 - UFSC-Pt116 + UFSC-Pt188, T7 - UFSC-SA9 + UFSC-Pt188 e T8 - UFSC-Pt116 + UFSC-Pt188 + UFSC-SA9. Cada tratamento foi composto por 10 repetições (cada vaso com uma muda de eucalipto foi considerada uma repetição).

\section{Local dos experimentos e isolados micorrízicos}

O experimento foi realizado em casa de vegetação na área experimental do Departamento de Solos da Universidade Federal de Santa Maria - UFSM/RS. Nesse estudo, em casa de vegetação, as sementes de eucalipto foram semeadas em vasos, sendo cada vaso considerado uma unidade experimental. Cada um dos oito tratamentos utilizados nesse estudo foi composto por 10 repetições (10 vasos, cada vaso com uma muda de eucalipto cada, totalizando 80 unidades experimentais).

Os isolados de fungos ectomicorrízicos usados neste experimento são oriundos de áreas com plantações de Eucalyptus spp. e foram cedidos pelo Laboratório de Ectomicorrizas do Departamento de Microbiologia e Parasitologia da Universidade Federal de Santa Catarina - UFSC/SC; e multiplicados no Laboratório de Biologia e Microbiologia do Solo e Meio Ambiente do Departamento de Solos da UFSM. Os isolados utilizados nesse estudo estão descritos na Tabela 1. 
TABELA 1: Isolados de fungos ectomicorrízicos.

TABLE 1: Isolates of Ectomycorrhizal fungi.

\begin{tabular}{ccc}
\hline $\begin{array}{l}\text { Isolado } \\
\text { fúngico }\end{array}$ & Espécie de fungo ectomicorrizico & Hospedeiro \\
\hline UFSC-Pt116 & Pisolithus microcarpus (Cooke \& Massee) Cunn. & Eucalyptus sp. \\
UFSC-SA9 & Scleroderma flavidum E. Et. E. & $\begin{array}{c}\text { Eucalyptus camaldulensis Dehnh. } \\
\text { Eucalyptus dunni Maiden }\end{array}$ \\
\hline
\end{tabular}

Os isolados fúngicos utilizados nesse estudo foram escolhidos com base nos resultados obtidos por Souza (2003), Souza (2004), Mello (2006) e Bonnassis (2007), nos quais estes apresentaram maior eficiência na acumulação de nutrientes e na promoção de crescimento das plantas de eucalipto.

As culturas puras destes fungos foram originalmente obtidas de carpóforos coletados em plantações de Eucalyptus spp. e são preservadas através de repicagens periódicas para meio de cultura Melin-Norkrans Modificado sólido (MNM) (MARX, 1969), em placas de Petri, acondicionadas em câmara incubadora B.O.D. a $25^{\circ} \mathrm{C}$. Cada isolado foi multiplicado a partir das culturas da coleção para meio de cultivo de mesma composição sob condições assépticas na câmara de fluxo laminar. As placas foram incubadas a 25 ${ }^{\circ} \mathrm{C}$ em estufa até a utilização das culturas, 30 dias após as multiplicações.

\section{Produção do inóculo de fungos ectomicorrízicos}

Para inocular os fECM às plantas de Eucalyptus grandis utilizou-se uma mistura com uma parte de vermiculita com granulometria média e quatro de turfa (v/v). A mistura vermiculita-turfa (1:4) foi previamente esterilizada em autoclave a $121^{\circ} \mathrm{C}$ e $1 \mathrm{~atm}$, durante $60 \mathrm{~min}$, (esta operação foi repetida por três dias consecutivos). Posteriormente, adicionou-se $200 \mathrm{~mL}$ de meio de cultura MNM (MARX, 1969) e foi realizada nova esterilização, pelo período de $20 \mathrm{~min}$, sob as mesmas condições de temperatura e pressão.

O inóculo dos fECM foi obtido em placas de Petri a partir de cultivo em meio de cultura MNM. Posteriormente, foram realizadas suspensões micelianas em $25 \mathrm{~mL}$ de MNM em erlenmeyers de $250 \mathrm{~mL}$, a partir de discos de $8 \mathrm{~mm}$ de diâmetro obtidos das culturas em placa, para posterior incubação a $25^{\circ} \mathrm{C}$, durante 30 dias.

Após o período de incubação, adicionou-se ao inóculo, $200 \mathrm{~mL}$ de MNM, para posterior fragmentação em liquidificador de alumínio esterilizado, durante 5 segundos. Este conteúdo foi utilizado para inocular $500 \mathrm{~mL}$ da mistura de vermiculita-turfa, a qual posteriormente foi armazenada para crescimento miceliano em frascos de vidraria (tipo conserva) de $900 \mathrm{~mL}$ a $25^{\circ} \mathrm{C}$ durante 90 dias.

\section{Solo}

O solo utilizado neste estudo foi o Neossolo Quartzarênico. As amostras de solo foram coletadas em uma área degradada, localizada no Município de São Francisco de Assis, região sudoeste do RS.

$\mathrm{O}$ solo foi previamente esterilizado em autoclave a $121^{\circ} \mathrm{C}$ durante 60 min três vezes, com um intervalo de $24 \mathrm{~h}$ entre cada esterilização, para serem eliminadas possíveis contaminações por outros fungos. $\mathrm{O}$ solo apresentou as seguintes características químicas após o processo de autoclavagem: $\mathrm{pH}=4,7 ; \mathrm{P}=$ 2,46 mg.dm ${ }^{-3} ; \mathrm{K}=10,6 \mathrm{mg} \cdot \mathrm{dm}^{-3} ; \mathrm{Al}=72 \%, \mathrm{Ca}=0,1 \mathrm{cmol}_{\mathrm{c}} \mathrm{dm}^{3}, \mathrm{Mg}=0,1 \mathrm{cmol}_{\mathrm{c}} \mathrm{dm}^{3}, \mathrm{CTC}=0,73 \mathrm{cmol}_{\mathrm{c}}$ $\mathrm{dm}^{3}$, M.O. $=0,25 \%$ e saturação por bases $=14 \%$. Todas as análises foram realizadas de acordo com os procedimentos da Comissão de Química e Fertilidade do Solo (CQFS, 2004). Após a esterelização, foi adicionada a este substrato uma solução nutritiva de: $\mathrm{Mn}, 0,15 ; \mathrm{Zn}, 0,0375 ; \mathrm{Cu}, 0,125 ; \mathrm{Mo}, 0,05 ; \mathrm{B}, 0,05$ e $\mathrm{Fe}, 0,375\left(\mathrm{mg} \mathrm{kg}^{-1}\right)$, baseada em resultados encontrados por Alves et al. (2001).

$\mathrm{O} \mathrm{N}$ e o $\mathrm{K}$ foram adicionados na forma de $\left(\mathrm{NH}_{4}\right)_{2} \mathrm{SO}_{4}$ e $\mathrm{KCl}$, respectivamente, no momento da semeadura, conforme a recomendação do Manual de Adubação e de Calagem para os Estados do RS e de SC (COMISSÃO DE QUÍMICA E FERTILIDADE DO SOLO, 2004). 


\section{Sementes}

As sementes de Eucalyptus grandis foram obtidas na Bolsa de Sementes do Laboratório de Silvicultura do Departamento de Ciências Florestais da UFSM, que, por sua vez, compra as sementes da Empresa IBEF, cuja produção é realizada em éreas de produção de sementes em Piracicaba - SP. As sementes foram coletadas no ano de 2009. Estas sementes foram pré-germinadas (cinco dias em placa de Petri), em uma incubadora microbiológica com fotoperíodo de 8 horas diárias de luz. As sementes foram inicialmente desinfestadas em álcool $70 \%$ por 30 segundos, lavadas em água destilada, e transferidas para placas de Petri contendo papel tipo toalha e água estéril (2,5 vezes o peso do papel).

\section{Inoculação dos fungos}

Após três meses de incubação do inóculo, foi realizada a inoculação dos isolados Pisolithus microcarpus (UFSC-Pt116), Scleroderma flavidum (UFSC-SA9) e Pisolithus microcarpus (UFSCPt188), em vasos de PVC, previamente desinfestados com solução de hipoclorito de sódio (1\%), com volume de $1 \mathrm{~L}$. A inoculação foi feita no momento da semeadura, colocando-se $10 \%$ (v/v) do inoculante fúngico (tratamentos) em cada vaso e o restante do volume do vaso foi preenchido com o solo neossolo quartzarênico. Nas misturas, combinou-se o inóculo dos isolados de modo a obter combinações de dois ou três isolados em cada vaso de plantio (Tabela 2). O tratamento-controle (não inoculado) recebeu os mesmos $10 \%(\mathrm{v} / \mathrm{v})$ do substrato de inoculação, porém, sem os fECM. A homogeneização do inóculo foi realizada manualmente. Cada vaso recebeu quatro sementes pré-germinadas de eucalipto. A umidade do solo foi elevada à capacidade de campo.

Após a inoculação e semeadura, o experimento foi mantido em casa de vegetação por 90 dias. A umidade na capacidade de campo foi mantida diariamente com a adição de água destilada. Passados 10 dias da emergência das plântulas, foi realizado o primeiro desbaste, deixando-se duas plântulas por vaso. Decorrido 20 dias da emergência, foi realizado um novo desbaste, deixando-se apenas uma planta de eucalipto por vaso.

\section{Avaliações}

Durante o crescimento, foram realizadas avaliações quinzenais quanto à altura e diâmetro do caule das mudas de eucalipto. Aos 60 dias após a semeadura, foram coletadas cinco plantas de cada tratamento, e analisadas quanto à altura $(\mathrm{cm})$, diâmetro do caule $(\mathrm{mm})$, massa seca das raízes $(\mathrm{mg} / \mathrm{planta})$, volume de raízes $\left(\mathrm{cm}^{3}\right)$, associação micorrízica (\%), massa seca da parte aérea (mg/planta) e teores $\mathrm{N}, \mathrm{P}$ e K na parte aérea das plantas. A mesma avaliação foi realizada aos 90 dias, com as cinco plantas restantes de cada tratamento.

No momento da coleta as plantas foram seccionadas em raízes e parte aérea. As raízes foram lavadas com auxílio de peneiras de $5 \mathrm{~mm}$. A parte aérea foi colocada em estufa a $70^{\circ} \mathrm{C}$ até atingir peso constante, para determinação da massa seca. A determinação da percentagem de colonização radicular foi realizada pela técnica das interseções de Giovannetti e Mosse (1980), modificado por Brundrett et al. (1996). Posteriormente, as raízes foram secas, conforme metodologia utilizada para a parte aérea. Na determinação dos teores de $\mathrm{P}$ e $\mathrm{K}$ nos tecidos das plantas, empregou-se a técnica descrita por Tedesco et al. (1995). O volume radicular foi determinado pelo método de deslocamento de água, adaptando-se metodologia utilizada para solos (EMBRAPA, 1997), anterior à secagem das raízes.

Os resultados foram testados quanto à sua normalidade e submetidos à análise de variância e, após, submetidos ao teste múltiplo de médias (Tukey, 5\%), utilizando-se os procedimentos disponíveis no programa estatístico SISVAR (FERREIRA, 2011).

\section{RESULTADOS E DISCUSSÃO}

Durante a condução desse estudo, os valores de altura de plantas variaram entre 2,7 e $46 \mathrm{~cm}$, aos 30 e aos 90 dias, respectivamente, após a semeadura (Tabela 2). A inoculação com o isolado de fECM UFSC- 
Pt116 favoreceu o crescimento em altura das plantas até os 45 dias após a semeadura sendo estatisticamente superior aos demais tratamentos aos 30 e 45 dias (Tabela 2). Aos 30 dias após a semeadura, o tratamento T2 atingiu $3,7 \mathrm{~cm}$ de altura, $37 \%$ superior ao controle. Nesse mesmo período, este tratamento foi seguido por grupo de plantas com valores semelhantes, que incluem os tratamentos T3, T4, T5 e T6. Nessa mesma avaliação os tratamentos T7 e T8 não apresentaram diferenças estatísticas em relação ao controle. Estes resultados corroboram os de Alves et al. (2001), que encontraram maiores valores de altura de plantas de Eucalyptus dunnii Maiden com a inoculação de fECMs do gênero Pisolithus sp.

$\mathrm{Na}$ avaliação aos 45 dias, as plantas do tratamento-controle obtiveram $5,4 \mathrm{~cm}$ de altura, enquanto que o tratamento inoculado com o isolado UFSC-Pt116 obteve $8,2 \mathrm{~cm}, 51 \%$ superior (Tabela 2). Isto pode ter ocorrido porque o isolado UFSC-Pt116 tem crescimento mais rápido que os demais isolados, e é eficiente em colonizar raízes, como constatado por Mello (2006), além de ser um formador natural de simbiose com o Eucalyptus grandis. Dos 60 aos 90 dias após a semeadura, não foram observadas diferenças significativas entre os tratamentos. O incremento nos valores de altura ao longo do tempo foi regular, até os 60 dias, apresentando $120 \%$ de crescimento a cada 15 dias. Dos 60 aos 75 dias o incremento de altura foi de 100\%; e, dos 75 aos 90 dias foi de $41 \%$. Do ponto de vista do tempo em que as plantas permanecem no viveiro até irem ao campo, a inoculação com fECM é interessante, pois, observando-se a Tabela 2 é possivel verificar que em um mesmo período todas as plantas inoculadas tiveram vantagens em relação à altura de plantas em relação ao controle, em especial aquelas inoculadas com o isolado UFSC-Pt188. Dessa forma, plantas que crescem melhor no viveiro também estão mais bem preparadas e desenvolvidas para o transplante no campo, o que resulta em melhores taxas de sobrevivência em regiões desfavoráveis como é o caso da região estudada nesse trabalho.

Os valores de diâmetro do caule variaram entre 1,0 a 4,5 mm, aos 30 e aos 90 dias após a semeadura (Tabela 3). Em relação ao diâmetro do caule das plantas, verificaram-se diferenças estatísticas significativas entre os tratamentos com inoculação individual e com misturas de isolados ectomicorrízicos, nas avaliações realizadas aos 60 e 75 dias.

TABELA 2: Altura das plantas de Eucalyptus grandis produzidas em Neossolo Quartzarênico, inoculadas com isolados de fungos ectomicorrízicos individualmente ou em mistura e controle, aos 30, 45, 60, 75 e 90 dias após a semeadura, em casa de vegetação.

TABLE 2: Eucalyptus grandis height produced in a Quartzarenic Neosols, inoculated with isolates of ectomycorrhizal fungi alone or in mixture and control, at 30, 45, 60, 75 and 90 days after planting, in the greenhouse.

\begin{tabular}{lccccc}
\hline \multirow{2}{*}{ Tratamentos } & \multicolumn{5}{c}{ Altura (cm) } \\
\cline { 2 - 6 } & \multicolumn{5}{c}{ Dias após a semeadura } \\
\cline { 2 - 6 } & 30 & 45 & 60 & 75 & 90 \\
\hline T1 - Controle & $2,7 \mathrm{cD}$ & $5,4 \mathrm{cD}$ & $13,9 \mathrm{abC}$ & $28,8 \mathrm{abB}$ & $38,6 \mathrm{aA}$ \\
T2 - UFSC-Pt116 & $3,7 \mathrm{aD}$ & $8,2 \mathrm{aD}$ & $17,4 \mathrm{aC}$ & $27,3 \mathrm{abB}$ & $40,7 \mathrm{aA}$ \\
T3 - UFSC-SA9 & $3,2 \mathrm{abD}$ & $7,1 \mathrm{abD}$ & $17,0 \mathrm{aC}$ & $33,6 \mathrm{abB}$ & $44,1 \mathrm{aA}$ \\
T4 - UFSC-Pt188 & $3,1 \mathrm{abD}$ & $6,8 \mathrm{abcD}$ & $17,3 \mathrm{aC}$ & $36,0 \mathrm{aB}$ & $46,0 \mathrm{aA}$ \\
T5 - UFSC-Pt116 + UFSC-SA9 & $3,2 \mathrm{abD}$ & $7,0 \mathrm{abD}$ & $15,4 \mathrm{abC}$ & $32,0 \mathrm{abB}$ & $42,9 \mathrm{aA}$ \\
T6 - UFSC-Pt116 + UFSC-Pt188 & $3,1 \mathrm{abD}$ & $7,1 \mathrm{abD}$ & $14,0 \mathrm{abC}$ & $29,5 \mathrm{abB}$ & $43,9 \mathrm{aA}$ \\
T7 - UFSC-SA9 + UFSC-Pt188 & $3,0 \mathrm{cE}$ & $6,7 \mathrm{abcD}$ & $15,2 \mathrm{abC}$ & $31,2 \mathrm{abB}$ & $43,7 \mathrm{aA}$ \\
T8 - UFSC-Pt116 + UFSC-Pt188 + UFSC-SA9 & $2,9 \mathrm{cC}$ & $5,8 \mathrm{bcC}$ & $10,5 \mathrm{bC}$ & $22,1 \mathrm{cB}$ & $39,9 \mathrm{aA}$ \\
\hline \multicolumn{1}{c}{ CV (\%) } & 13,8 & 15,6 & 17,8 & 21,5 & 18,2 \\
\hline
\end{tabular}

Em que: Médias seguidas das mesmas letras minúsculas nas colunas e por letras maiúsculas nas linhas não diferem estatisticamente entre si pelo teste de Tukey a 5\%. Sendo: UFSC-Pt116 - Pisolithus microcarpus; UFSC-SA9 Scleroderma flavidum; UFSC-Pt188 - P. microcarpus 
Aos 60 dias após a semeadura, as plantas do tratamento T2, inoculadas com o isolado UFSCPt116, obtiveram diâmetro do caule estatisticamente superiores ao controle (Tabela 3). Já aos 75 dias, as plantas do tratamento T4, inoculadas com o isolado UFSC-Pt188, destacaram-se das demais. Esses resultados corroboram os observados por Alves et al. (2001), que verificaram valores de diâmetro do caule superiores em plantas de eucalipto inoculadas com fECM em relação às plantas-controle. Já aos 90 dias após a semeadura, as plantas não apresentaram diferenças estatísticas significativas entre si para esta variável. Observa-se ainda que, aos 60 e aos 75 dias, os tratamentos que obtiveram os maiores valores de diâmetro do caule foram aqueles com inoculação individual dos isolados de fungos ectomicorrízicos. Os resultados positivos da inoculação dos isolados UFSC-Pt116, aos 60 dias, e, do UFSC-Pt188, aos 75 dias, se devem ao fato dos isolados colonizarem com eficiência as raízes do eucalipto, como foi constatado por Mello (2006) e por Bonnassis (2007), em seus estudos, que avaliaram a inoculação fECMs em Eucalyptus grandis e Eucalyptus dunnii, respectivamente. Além disso, no trabalho de Souza (2003) foi observado que, enquanto alguns isolados de fECM contribuíam para uma maior acumulação de biomassa na parte aérea de plantas, outros promoviam uma maior acumulação de P. Isso pode ter ocorrido também nesse estudo, pois em alguns tratamentos o UFSC-Pt116 inoculado sozinho e em mistura teve melhor resultado em relação ao diametro do caule e altura de plantas, enquanto que em outros tratamentos com o UFSC-Pt188 inoculado sozinho e em mistura, o resultado melhor foi em relação ao acúmulo de nutientes como o P. Do ponto de vista de melhor crescimento desenvolvimento no viveiro e posteriormente no campo, a inoculação com misturas de mais de um isolado é importante, pois permite que as plantas não só cresçam melhor, mas também acumulem reservas de nutrientes, importantes para o período de adaptação após o transplante no campo em regiões que apresentam solos com deficiencias nutricionais como as áreas passíveis de processos de arenização, como as estudadas nesse trabalho.

$\mathrm{O}$ incremento do diâmetro do caule ao longo do período avaliado não foi regular (Tabela 3). Não houve diferenças em relação ao diâmetro do caule entre 30 e 45 dias após a semeadura. Dos 45 até os 60 dias ocorreu um incremento de $120 \%$ no diâmetro do caule das plantas (média geral de 1,0 mm para 2,2

TABELA 3: Diâmetro do caule das plantas de Eucalyptus grandis produzidas em Neossolo Quartzarênico, inoculadas e não inoculadas com diferentes isolados de fECM individualmente ou em mistura, aos 30, 45, 60, 75 e 90 dias após a semeadura em casa de vegetação.

TABLE 3: Stem diameter of Eucalyptus grandis trees produced in Quartzarenic Neosols, non-inoculated and inoculated with fECM alone or in mixture, at 30, 45, 60, 75 and 90 days after planting, in the greenhouse.

\begin{tabular}{lccccc}
\hline \multirow{2}{*}{ Tratamento } & \multicolumn{5}{c}{ Diâmetro do caule (mm) } \\
\cline { 2 - 6 } & \multicolumn{5}{c}{ Dias após a semeadura } \\
\cline { 2 - 6 } & 30 & 45 & 60 & 75 & 90 \\
\hline T1 - Controle & $1,0 \mathrm{aC}$ & $1,0 \mathrm{aC}$ & $1,6 \mathrm{bC}$ & $2,5 \mathrm{cB}$ & $3,5 \mathrm{aA}$ \\
T2 - UFSC-Pt116 & $1,0 \mathrm{aC}$ & $1,0 \mathrm{aC}$ & $3,0 \mathrm{aB}$ & $3,1 \mathrm{abcB}$ & $4,1 \mathrm{aA}$ \\
T3 - UFSC-SA9 & $1,0 \mathrm{aD}$ & $1,0 \mathrm{aD}$ & $2,4 \mathrm{abC}$ & $3,3 \mathrm{abcB}$ & $4,5 \mathrm{aA}$ \\
T4 - UFSC-Pt188 & $1,0 \mathrm{aD}$ & $1,0 \mathrm{aD}$ & $2,5 \mathrm{abC}$ & $4,0 \mathrm{aB}$ & $4,5 \mathrm{aA}$ \\
T5 - UFSC-Pt116 + UFSC-SA9 & $1,0 \mathrm{aD}$ & $1,0 \mathrm{aD}$ & $2,6 \mathrm{abC}$ & $3,6 \mathrm{abcB}$ & $4,3 \mathrm{aA}$ \\
T6 - UFSC-Pt116+ UFSC-Pt188 & $1,0 \mathrm{aD}$ & $1,0 \mathrm{aD}$ & $2,4 \mathrm{abC}$ & $3,6 \mathrm{abcB}$ & $4,4 \mathrm{aA}$ \\
T7 - UFSC-SA9 + UFSC-Pt188 & $1,0 \mathrm{aD}$ & $1,0 \mathrm{aD}$ & $2,2 \mathrm{abC}$ & $3,8 \mathrm{abB}$ & $4,3 \mathrm{aA}$ \\
T8 - UFSC-Pt116 + UFSC-Pt188+ UFSC-SA9 & $1,0 \mathrm{aD}$ & $1,0 \mathrm{aD}$ & $1,5 \mathrm{bC}$ & $2,8 \mathrm{bcB}$ & $3,8 \mathrm{aA}$ \\
\hline \multicolumn{1}{c}{ CV (\%) } & 0,0 & 0,0 & 23,8 & 16,1 & 14,1 \\
\hline
\end{tabular}

Em que: Médias seguidas das mesmas letras minúsculas nas colunas e por letras maiúsculas nas linhas não diferem estatisticamente entre si pelo teste de Tukey a 5\%. Sendo: UFSC-Pt116 - Pisolithus microcarpus; UFSC-SA9 Scleroderma flavidum; UFSC-Pt188 - Pisolithus microcarpus. 
$\mathrm{mm}$ ), mas com diferença apenas no tratamento em que as plantas foram inoculadas com o isolado UFSCPt116. Já entre 60 e 75 dias apresentaram 50\% de incremento, com alguns isolados apresentando diferenças, enquanto que nos últimos 15 dias, o incremento foi de $24 \%$. Embora dois tratamentos tenham apresentado diferenças significativas entre si, Souza (2003) considera que as medidas de diâmetro do caule têm pouca utilidade para avaliar o efeito da inoculação nesta fase do desenvolvimento das plantas. Assim, os valores de massa seca são considerados as variáveis mais úteis para medir o efeito de tratamentos de inoculação micorrízica sobre o crescimento das plantas (MARX, 1991).

Os valores de massa seca da parte aérea de plantas variaram entre 186 e $4.828 \mathrm{mg}$ planta $^{-1}$ aos 60 (T1 - controle) e aos 90 dias (T5 - UFSC-Pt116 + UFSC-SA9) após a semeadura (Tabela 4). Para a massa seca da parte aérea das plantas de eucalipto, aos 60 dias, T2 - UFSC-Pt 116 foi estatisticamente superior aos tratamentos T8 e ao controle, sendo 3,2 vezes superior ao controle. Os maiores valores de massa seca da parte aérea observados no tratamento inoculado com o isolado UFSC-Pt116 aos 60 dias, está relacionado com a maior altura e diâmetro do caule que foram verificados neste mesmo período. Estes resultados corroboram com os de Silva et al. (2007), que observaram maiores valores de massa seca em plantas de Eucalyptus grandis inoculadas com fECMs, quando comparados com plantas-controle. Estes resultados evidenciam a eficiência de alguns isolados fúngicos em promover maior acúmulo de massa seca em plantas de eucalipto, além de proteger contra estresses e auxiliar na absorção de água e nutrientes, quando em condições de campo. Estes resultados corroboram os obtidos por Souza et al. (2008), que ao trabalhar com novos isolados de fECMs no crescimento de eucalipto, verificaram maiores valores de massa seca em plantas inoculadas, quando comparadas com as plantas-controle.

Com relação à massa seca de parte aérea aos 90 dias, não foram observadas diferenças significativas entre os tratamentos, com exceção do tratamento $\mathrm{T} 8$, que foi significativamente inferior a alguns tratamentos (Tabela 4). Neste caso, o tratamento com a inoculação da mistura de alguns isolados provavelmente causou

TABELA 4: Massa seca da parte aérea e de raízes das plantas de Eucalyptus grandis produzidas em Neossolo Quartzarênico, inoculadas com diferentes isolados de fungos ectomicorrízicos individualmente ou em mistura e o controle, aos 60 e 90 dias após a semeadura, em casa de vegetação.

TABLE 4: Dry mass of shoots and roots of Eucalyptus grandis produced in a Quartzarenic Neosols, inoculated with different isolates of ectomycorrhizal fungi alone or in mixture, at 60 and 90 days after planting in the greenhouse.

\begin{tabular}{|c|c|c|c|c|}
\hline \multirow{4}{*}{ Tratamento } & \multicolumn{4}{|c|}{ Massa seca (mg planta $\left.{ }^{-1}\right)$} \\
\hline & \multicolumn{2}{|c|}{ Parte aérea } & \multicolumn{2}{|c|}{ Raízes } \\
\hline & \multicolumn{4}{|c|}{ Dias após a semeadura } \\
\hline & 60 dias & 90 dias & 60 dias & 90 dias \\
\hline T1 - Controle & $186 \mathrm{cB}$ & $3.360 \mathrm{abA}$ & $81 \mathrm{abB}$ & $926 \mathrm{abA}$ \\
\hline T2 - UFSC-Pt116 & $608 \mathrm{aB}$ & $4.792 \mathrm{aA}$ & $113 \mathrm{aB}$ & $1.127 \mathrm{abA}$ \\
\hline T3 - UFSC-SA9 & $391 \mathrm{abcB}$ & $4.512 \mathrm{aA}$ & $82 \mathrm{abB}$ & $1.065 \mathrm{abA}$ \\
\hline T4 - UFSC-Pt188 & $278 \mathrm{abcB}$ & $4.057 \mathrm{aA}$ & $64 \mathrm{abB}$ & $1.178 \mathrm{aA}$ \\
\hline T5 - UFSC-Pt116 + UFSC-SA9 & $377 \mathrm{abcB}$ & $4.828 \mathrm{aA}$ & $61 \mathrm{abB}$ & $1.058 \mathrm{abA}$ \\
\hline T6 - UFSC-Pt116 + UFSC-Pt188 & $457 \mathrm{abB}$ & $4.405 \mathrm{aA}$ & $59 \mathrm{abB}$ & $8.14 \mathrm{abA}$ \\
\hline T7 - UFSC-SA9 + UFSC-Pt188 & $396 \mathrm{abcB}$ & $3.978 \mathrm{abA}$ & $66 \mathrm{abB}$ & $1.063 \mathrm{abA}$ \\
\hline T8 - UFSC-Pt116 + UFSC-Pt188 + UFSC-SA9 & $199 \mathrm{bcB}$ & $2.176 \mathrm{bA}$ & $31 \mathrm{bB}$ & $678 \mathrm{bA}$ \\
\hline $\mathrm{CV}(\%)$ & 34,5 & 21,7 & 40,7 & 23,7 \\
\hline
\end{tabular}

Em que: Médias seguidas das mesmas letras nas colunas e por letras maiúsculas nas linhas para parte aérea e raízes não diferem estatisticamente entre si pelo teste de Tukey a 5\%. Sendo: UFSC-Pt116 - Pisolithus microcarpus; UFSCSA9 - Scleroderma flavidum; UFSC-Pt188 - Pisolithus microcarpus. 


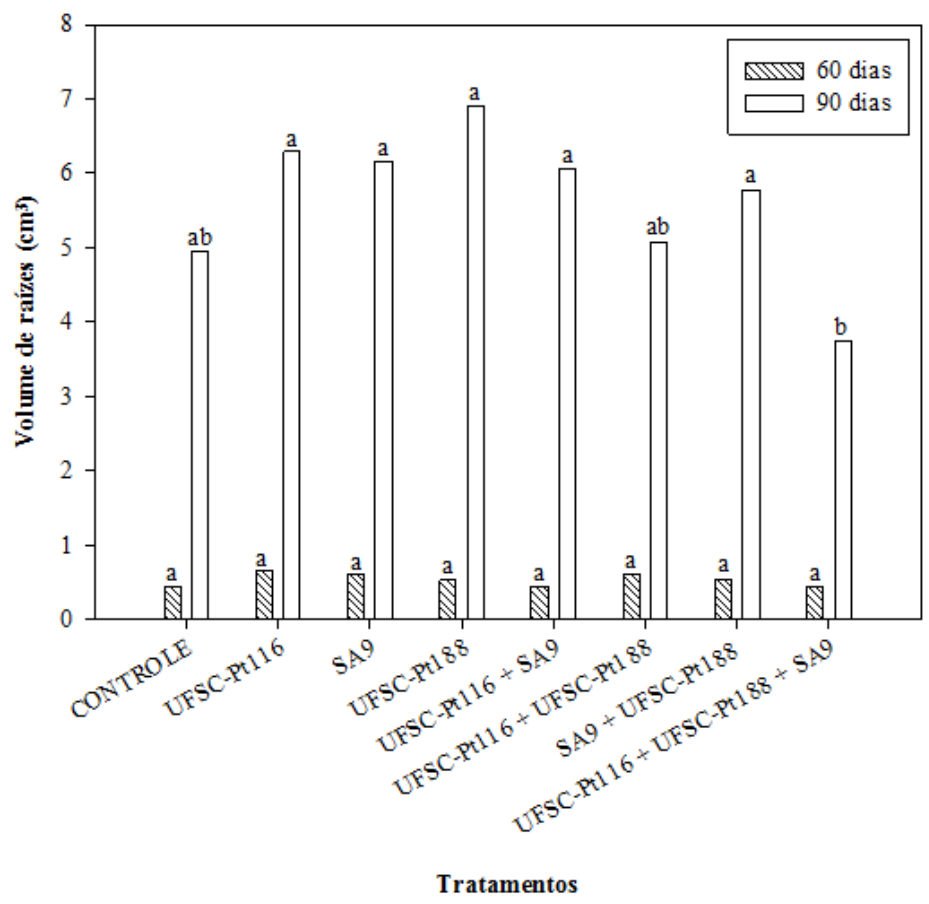

FIGURA 1: Volume de raízes das plantas de Eucalyptus grandis produzidas em Neossolo Quartzarênico, inoculadas ou não com diferentes isolados de fungos ectomicorrízicos individualmente ou em mistura, aos 60 e 90 dias após o plantio em casa de vegetação. Médias seguidas das mesmas letras nas colunas (tratamentos) não diferem estatisticamente entre si pelo teste de Tukey a 5\%. UFSC-Pt116 - Pisolithus microcarpus; UFSC-SA9 - Scleroderma flavidum; UFSC-Pt188 - P. microcarpus

FIGURE 1: Toots volume of Eucalyptus grandis produced in a Quartzarenic Neosols, or non-inoculated with different isolates of ectomycorrhizal fungi alone or in mixture, at 60 and 90 days after planting in the greenhouse. Means followed by same letters in columns do not differ among themselves by Tukey test at $5 \%$.

efeito não benéfico para as plantas, no que diz respeito ao incremento de massa seca da parte aérea. Isso pode ocorrer naturalmente, pois existem ectomicorrizas que formam associação em fases mais juvenis da planta e outras ectomicorrizas que formam associação em fases posteriores, ocorrendo uma mudança natural na espécie e ectomicorriza que se associa com a planta ao longo do crescimento. Portanto, a inoculação dos isolados em mistura pode ter causado competição por sítios de formação de associação micorrízica com a planta.

Os valores de massa seca de raízes das plantas variaram entre 31 (T8) e $1.178 \mathrm{mg}$ planta $^{-1}$ (T40, aos 60 e aos 90 dias após a semeadura, respectivamente (Tabela 4). Aos 60 e 90 dias não foram observadas diferenças estatísticas significativas entre os tratamentos e as plantas-controle, com exceção do T8, que obteve valores inferiores ao T2 aos 60 dias e ao T4 aos 90 dias. Aos 60 e 90 dias, o tratamento UFSCPt 116 se destacou dos demais, com 113 e $1.127 \mathrm{mg}$, ou seja, aproximadamente 40 e $20 \%$ superior ao controle, respectivamente. Os maiores valores de massa seca da parte aérea, observados nos tratamentos com o isolado UFSC-Pt116 aos 60 dias podem ser resultado da maior altura e diâmetro do caule que foram observados nas plantas neste mesmo período. Estes resultados são semelhantes aos obtidos por Silva et al. (2007) e Souza (2004), que encontraram maiores valores de massa seca em plantas de Eucalyptus grandis inoculadas com isolados fúngicos ectomicorrízicos, quando comparados com o controle.

Em relação ao volume de raízes, aos 60 dias após a semeadura, não foram observadas diferenças estatísticas entre os tratamentos (Figura 1). 
Já aos 90 dias após a semeadura, o volume de raízes do tratamento T 8 - UFSC-Pt116 + UFSCPt188 + SA9 foi estatisticamente inferior a todos os tratamentos, com exceção ao T1 - controle. Nesses tratamentos com maior volume de raízes, a associação ectomicorrízica com a planta estimulou o crescimento das raízes, o que pode ocorrer através da produção de hormônios pelos fungos micorrízicos que promovem esse estímulo. Segundo Silva et al. (2007), espera-se maior capacidade de absorção de nutrientes e água pelas plantas micorrizadas, também observado neste trabalho para o parâmetro volume de raízes.

Os isolados de fungos ectomicorrízicos colonizaram as raízes de eucalipto, produzidas no Neossolo, com diferente grau de intensidade, variando com o tipo de mistura que foi inoculado nas plantas (Figura 2). Aos 60 dias após a emêrgencia das plântulas, foi verificado que as plantas que obtiveram as maiores taxas de colonização foram as dos tratamentos UFSC-Pt116 e UFSC-SA9, com aproximadamente $20 \%$ de colonização radicular, seguidas de um segundo grupo composto pelas plantas dos demais tratamentos (UFSC-Pt188, UFSC-Pt116 + UFSC-SA9, UFSC-Pt116 + UFSC-Pt188, UFSC-SA9 + UFSC-Pt188 e UFSC-Pt116 + UFSC-Pt188 + UFSC-SA9). Apenas o controle não apresentou colonização micorrízica. Todas as plantas que foram inoculadas com fECMs apresentaram colonização radicular estatisticamente superior ao controle.

Aos 90 dias após a semeadura, os tratamentos seguiram a mesma têndencia em relação à colonização micorrízica, apresentando diferenças estatísticas entre si, mas com incremento de aproximadamente $3,3 \%$ em relação a primeira avaliação (60 dias), com exceção do controle que continuou sem colonização micorrízica (Figura 2). O incremento da colonização micorrízica pode ter sido influenciado

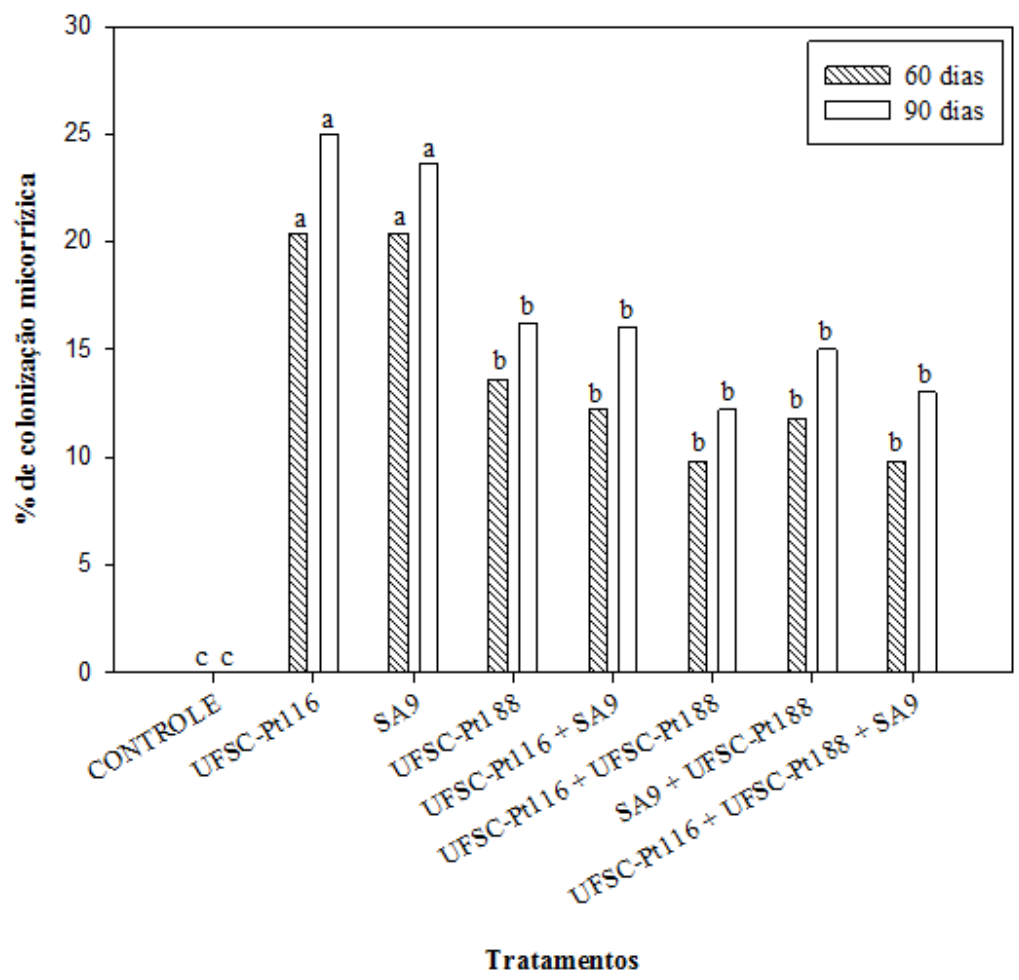

FIGURA 2: Colonização micorrízica nas plantas de Eucalyptus grandis produzidas em Neossolo Quartzarênico, inoculadas ou não com diferentes isolados de fungos ectomicorrízicos individualmente ou em mistura, aos 60 e 90 dias após o plantio em casa de vegetação. Médias seguidas das mesmas letras nas colunas não diferem estatisticamente entre si pelo teste de Tukey a $5 \%$.

FIGURE 2: Mycorrhizal colonization of Eucalyptus grandis plants produced in a Quartzarenic Neosols, or not inoculated with different isolates of ectomycorrhizal fungi alone or in mixture, at 60 and 90 days after planting in the greenhouse. Means followed by same letters in columns do not differ among themselves by Tukey test at $5 \%$. 
pelo tipo de solo utilizado no experimento, pois o Neossolo Quartzarênico é um solo frágil, com estrutura deficiente e com fertilidade natural baixa, o que pode ter favorecido a ocorrência da simbiose nos tratamentos com inoculação dos isolados fúngicos. Embora a colonização micorrízica obtida neste trabalho seja inferior à obtida por Alves et al. (2001), mesmo com uma baixa colonização, os isolados UFSC-Pt116 e UFSC-SA9 foram aqueles que apresentaram os maiores efeitos benéficos sobre as plantas, na maioria dos parâmetros avaliados nesse estudo. Os resultados obtidos nesse estudo em relação à colonização micorrízica são inferiores aos obtidos por Mello (2006), que trabalhou com substrato turfa, mais rico em nutrientes, por 120 dias em casa de vegetação.

Os tratamentos apresentaram diferenças significativas em relação ao teor de nutrientes na parte aérea das plantas aos 60 dias para o $\mathrm{N}$ e o $\mathrm{P}$, e aos 90 dias para o $\mathrm{N}$ e o $\mathrm{P}$ em relação ao controle (Tabela 5). Para o K não foram observadas diferenças significativas entre os tratamentos inoculados e o controle em nenhuma das avaliações.

Aos 60 dias após a semeadura, percentagem de $\mathrm{N}$ nas plantas de todos no tratamento T5 (UFSCPt116 + UFSC-SA9) foi de 3,5\%, enquanto o controle apresentou 2,0\%. Já aos 90 dias, o tratamento T2 (UFSC-Pt116) apresentou diferença estatística em relação ao teor de nitrogênio quando comparado com o controle, atingindo $2,8 \%$ de $\mathrm{N}$ na parte aérea, enquanto que o controle obteve $1,0 \%$. O tratamento UFSCPt116 foi seguido pelo UFSC-Pt116 + UFSC-Pt188 + UFSC-SA9 e também pelo tratamento UFSC-SA9, com valores um pouco mais baixos, seguidos pelos demais tratamentos incluindo o controle, não diferindo estatisticamente entre si. Estes resultados reforçam a eficiência do isolado UFSC-Pt116 em promover maior acúmulo de nitrogênio quando inoculado em plantas de eucalipto.

Para o acúmulo de P na parte aérea das plantas, aos 60 dias, verificou-se que as plantas do tratamento T4, foram superiores ao controle em cerca de $25 \%$ superior ao controle $\left(2,2 \mathrm{~g} . \mathrm{kg}^{-1}\right)$. Já aos 90 dias, verificouse que o tratamento T8 (UFSC-Pt116 + UFSC-Pt188 + UFSC-SA9) alcançou maiores teores de P que o controle. Observa-se que o tratamento que obteve maior acúmulo de fósforo na parte aérea contém o isolado UFSC-Pt188 na sua mistura, e que para os teores de nutrientes dos resultados apresentados, os tratamentos que acumularam maiores quantidades de nutrientes foram aqueles em que se inoculou os isolados fúngicos em misturas, conforme mencionado na primeira etapa deste trabalho. É importante salientar, que o teor

TABELA 5: Teores de nutrientes na parte aérea das plantas de Eucalyptus grandis produzidas em Neossolo Quartzarênico, inoculadas e não inoculadas com diferentes isolados de fungos ectomicorrízicos individualmente ou em mistura, aos 60 e 90 dias após o plantio em casa de vegetação.

TABLE 5: Levels of nutrients in the shoots of Eucalyptus grandis produced in a sandy, inoculated and noninoculated with isolates of ectomycorrhizal fungi alone or in mixture, at 60 and 90 days after planting, in the greenhouse.

\begin{tabular}{ccccccc}
\hline & \multicolumn{3}{c}{$\mathrm{N}(\%)$} & \multicolumn{3}{c}{$\mathrm{P}\left(\mathrm{g} \mathrm{kg}^{-1}\right)$} \\
$\mathrm{K}\left(\mathrm{g} \mathrm{kg}^{-1}\right)$ \\
\cline { 2 - 7 } Tratamento & \multicolumn{5}{c}{ Dias após a semeadura } \\
\cline { 2 - 7 } & 20 & 90 & 60 & 90 & 60 & 90 \\
\hline T1 - Controle & $3,1 \mathrm{cA} *$ & $1,0 \mathrm{bB}$ & $2,2 \mathrm{bA}$ & $1,6 \mathrm{cA}$ & $21,2 \mathrm{abA}$ & $22,2 \mathrm{aB}$ \\
T2 - UFSC-Pt116 & $3,3 \mathrm{aA}$ & $1,7 \mathrm{abB}$ & $2,4 \mathrm{abA}$ & $1,6 \mathrm{cB}$ & $22,4 \mathrm{abA}$ & $22,1 \mathrm{aB}$ \\
T3 - UFSC-SA9 & $3,4 \mathrm{aA}$ & $1,2 \mathrm{bB}$ & $2,8 \mathrm{aA}$ & $1,8 \mathrm{bcA}$ & $23,6 \mathrm{aA}$ & $25,3 \mathrm{aB}$ \\
T4 - UFSC-Pt188 & $3,5 \mathrm{aA}$ & $1,1 \mathrm{bB}$ & $2,4 \mathrm{abA}$ & $2,4 \mathrm{abA}$ & $22,6 \mathrm{abA}$ & $24,8 \mathrm{aB}$ \\
T5 - UFSC-Pt116 + UFSC-SA9 & $3,2 \mathrm{abA}$ & $1,1 \mathrm{bB}$ & $2,3 \mathrm{bA}$ & $1,9 \mathrm{bcA}$ & $21,6 \mathrm{abA}$ & $25,8 \mathrm{aB}$ \\
T6 - UFSC-Pt116 + UFSC-Pt188 & $3,4 \mathrm{aA}$ & $1,1 \mathrm{bB}$ & $2,2 \mathrm{bA}$ & $2,0 \mathrm{abcA}$ & $21,6 \mathrm{abA}$ & $26,1 \mathrm{aB}$ \\
T7 - UFSC-SA9 + UFSC-Pt188 & $2,8 \mathrm{bA}$ & $2,0 \mathrm{abB}$ & $2,2 \mathrm{bB}$ & $2,8 \mathrm{aA}$ & $23,1 \mathrm{abA}$ & $25,6 \mathrm{aA}$ \\
\hline T8 - UFSC-Pt116 + UFSC-Pt188 + UFSC-SA9 & 6,5 & 44,1 & 8,6 & 18,1 & 5,7 & 11,5 \\
\hline CV (\%) & & &
\end{tabular}

Em que: Médias seguidas das mesmas letras nas colunas e por letras maiúsculas nas linhas entre 60 e 90 dias para cada nutriente não diferem estatisticamente entre si pelo teste de Tukey a $5 \%$. 
ótimo de P no solo para a máxima eficiência do fungo não é suficiente para a produção econômica das plantas. Soares (1986) cita em seu trabalho que os teores de $\mathrm{P}$ usados na formação das plantas de eucalipto em viveiro são altos e inibem a colonização do fungo, assim poderá não ocorrer a colonização micorrízica. Por este motivo, neste trabalho não foi adicionado $\mathrm{P}$ ao solo na instalação do experimento, no intuito de favorecer o estabelecimento da associação ectomicorrízica com as plantas de eucalipto.

Os resultados para os teores de P são semelhantes aos encontrados por Souza (2004), que verificou que as plantas de Eucalyptus dunnii acumularam maiores teores de $P$ na parte aérea quando foram inoculados com fECMss do gênero Pisolithus. Entretanto, Silva et al. (2007) não observaram diferenças significativas no teor de P na parte aérea de plantas de Eucalyptus grandis, quando essas foram inoculadas com o isolado UFSC-Pt116.

O conjunto de dados observados nesse estudo forneceram importantes informações no que diz respeito à inoculação de fECM em plantas de eucalipto em condições de casa de vegetação, pois foi possível observar que em determinados períodos alguns isolados parecem contribuir melhor para o crescimento inicial de plantas enquanto que outros contribuem melhor para a acumulação de nutrientes. Dessa forma, torna-se importante que a inoculação seja realizada com mais de um isolado de fECM, pois assim serão alcançados os dois tipos de vantagens citados, o que resultará em maior crescimento de plantas durante o período de viveiro, além da maior acumulação de nutrientes escassos como o P. Esse fatores em conjunto podem favorecer o estabelecimento das mudas, através de melhores taxas de sobrevivência e desenvolvimento, após o tranplante para essas áreas que estão sob processo de arenização no sudoeste do $\mathrm{RS}$, quando comparadas com mudas tradicionalmente produzidas nos viveiros.

Alguns dos parâmetros avaliados mostraram benefícios advindos da inoculação de isolados ectomicorrízicos em plantas de eucalipto em Neosssolo Quartzarênico, que pode ser favorável em situações em que se tenta fazer a recuperação e a utilização de solos pobres, degradados e com baixa fertilidade. Sendo assim, a inoculação com algumas espécies de fungos ectomicorrízicos pode ser uma alternativa para auxiliar no estabelecimento de plantas em áreas com essas características, pois poderá propiciar melhorias e benefícios no estabelecimento e crescimento das plantas, tornando-se uma estratégia que poderá ser utilizada na produção de mudas de eucalipto, destinadas para áreas em processo de arenização, como as que ocorrem ma metade sul do RS.

\section{CONCLUSÕES}

O uso dos isolados de fungos ectomicorrízicos UFSC-Pt116 - Pisolithus microcarpus; UFSC-SA9 - Scleroderma flavidum; UFSC-Pt188 - Pisolithus microcarpus individualmente, promovem maior altura, diâmetro de caule, acúmulo de massa seca da parte aérea e volume inicial de raízes em mudas de Eucalyptus grandis.

Os isolados ectomicorrízicos UFSC-Pt116 e UFSC-SA9 apresentam maior colonização radicular nas plantas de Eucalyptus grandis, em Neossolo Quartzarênico.

Os teores de fósforo na parte aérea das plantas de Eucalyptus grandis são maiores quando o eucalipto recebe tratamento inoculado com o isolado fúngicos UFSC-Pt188.

As plantas de Eucalyptus grandis apresentam maior teor nitrogênio na parte aérea quando inoculadas com o isolado fúngicos UFSC-Pt 116. A inoculação com mistura dos isolados fúngicos ectomicorrízicos UFSC-Pt116+UFSC-SA9+UFSC-Pt188 não promove melhor desenvolvimento nas plantas de eucalipto, em Neossolo Quartzarênico.

\section{AGRADECIMENTOS}

Os autores agradecem ao Programa de Pós-graduação em Ciência do Solo (PPGCS) da Universidade Federal de Santa Maria-UFSM, ao Conselho Nacional de Pesquisa e Tecnologia (CNPq), à Coordenação de Aperfeiçoamento de Pessoal de Nível Superior (CAPES) e à Fundação de Amparo à Pesquisa do estado do Rio Grande do Sul (FAPERGS) pelo apoio financeiro para execução do trabalho. 


\section{REFERÊNCIAS}

ALVES, J. R. et al. Efeito de inoculante ectomicorrízico produzido por fermentação semi-sólida sobre o crescimento de Eucalyptus dunnii Maiden. Pesquisa Agropecuária Brasileira, Brasília, v. 36, n. 2, p. 307-313, 2001.

ASSOCIAÇÃO BRASILEIRA DOS PRODUTORES DE FLORESTAS PLANTADAS. Anuário Estatístico da ABRAF 2011 ano base 2010. Brasília: ABRAF, 2011. 130 p.

BARROS, N. F.; BRANDI, R. M.; REIS, M. S. Micorriza em eucalipto. Uma revisão sobre a morfologia, a fisiologia e os efeitos mútuos da associação fungo-planta. Revista Árvore, Viçosa, MG, v. 2, n. 2, p. 130-140, 1978.

BONNASSIS, P. A. P. Caracterização de isolados fúngicos ectomicorrízicos na promoção do crescimento e na colonização radicular de Eucalyptus dunnii Maiden. 2007. 68 f. Dissertação (Mestrado) - Universidade Federal de Santa Catarina, Florianópolis, 2007.

BRUNDRETT, M. et al. Working with mycorrhizas in forestry and agriculture. Canberra: ACIAR, 1996. 374 p. (Monograph, 32).

COMISSÃO DE QUÍMICA E FERTILIDADE DO SOLO - RS/SC. Manual de adubação e calagem para os Estados do Rio Grande do Sul e de Santa Catarina. 10. ed. Porto Alegre: SBCS, Comissão de Química e Fertilidade do Solo, 2004. 394 p.

DORNELES, F. O. Produção de sementes de Lupinus albescens para recuperação de áreas degradadas por areização. 2009. 57 f. Dissertação (Mestrado) - Universidade Federal de Santa Maria, Santa Maria, 2009.

EMBRAPA. Dedicação à pesquisa florestal. Colombo: Embrapa Florestas, 2003. 54 p.

EMBRAPA. Manual de métodos de análise de solo. 2. ed. Rio de Janeiro: Centra Nacional de Pesquisas do Solo, 1997. 212 p.

EMBRAPA. Tecnologia de recuperação de áreas degradadas é exportada. Brasília, 2002. Disponível em: $<$ https://www.embrapa.gov.br/noticias $>$. Acesso em: 10 ago. 2015.

FERREIRA, D. F. Sisvar: A computer statistical analysis system. Ciência e Agrotecnologia, Lavras, v. 35, n. 6, p. 1039-1042, nov./dez. 2011.

FERREIRA, R. S. Tolerância de espécies florestais arbóreas e fungos ectomicorrízicos ao cobre. 2007. 135 f. Tese (Doutorado) - Universidade Federal de Santa Maria, Santa Maria, 2007. GARAY, I. et al. Evaluation of soil conditions in fast-growing plantations of Eucalyptus grandis and Acacia mangium in Brazil: a contribution to the study of sustainable land use. Applied Soil Ecology, Amsterdam, v. 27, n. 2, p. 177-187, 2004.

GIOVANNETTI, M.; MOSSE, B. An evaluation of techniques for measuring vesicular arbuscular mycorrhizal infection in roots. New Phytologist, Cambridge, v. 84, p. 489-500, 1980.

GLOWA, K. R.; AROCENA, J. M.; MASSICOTTE, H. B. Extraction of potassium and/or magnesium from selected soil minerals by Piloderma. Geomicrobiology Journal, Abingdon, v. 20, n. 2, p. 99-111, 2003.

LEITE, F. P. et al. Nutrient relations during an eucalyptus cycle at different population densities. Revista Brasileira de Ciência do Solo, Viçosa, MG, v. 35, p. 949-959, 2011.

MARX, D. H. The influence of ectotrophic mycorrhizal fungi on the resistance of pine roots to pathogenic infections. I. Antagonism of mycorrhizal fungi to root pathogenic fungi and soil bacteria. Phytopathology, Saint Paul, v. 59, n. 2, p. 153-163, 1969.

MARX, D. H. The practical significance of ectomycorrhizae in Forest establishment. In: THE MARCUS WALLENBERG FOUNDATION SYMPOSIUM, 1991, Stockolm. Proceedings... Wallenberg Foundation, 1991. p. 54-90.

MELLO, A. H. Ocorrência, caracterização e eficiência de fungos micorrízicos em Eucalyptus grandis e Acacia mearnsii. 2006. 236 f. Tese (Doutorado) - Universidade Federal de Santa Maria, Santa Maria, 2006. MOLLER, D. O. et al. Diagnóstico sobre a presença de areais na região Sudoeste do Rio Grande do Sul. Porto Alegre: SUDESUL, 1975.

RODRIGUES, F. A. V. et al. Disponibilidade de cobre para mudas de eucalipto em solos de cerrado. Revista Brasileira de Ciência do Solo, Viçosa, MG, v. 34, p. 1923-1932, 2010.

SANTOS, J. D. E. et al. Eficiência de fungos micorrízicos arbusculares isolados de solos de áreas de 
mineração de bauxita no crescimento inicial de espécies nativas. Revista Brasileira de Ciência do Solo, Viçosa, MG, v. 32, n. 1, p. 141-150, 2008.

SILVA, M. A. et al. Formação de ectomicorrizas por monocários e dicários de Pisolithus sp. e interações em Eucalyptus grandis. Revista Brasileira de ciência do Solo, Viçosa, MG, v. 31, n. 5, p. 917-929, 2007. SOARES, I. Níveis de fósforo no desenvolvimento de ectomicorrizas por Pisolithus tinctorius (Pers) Coker \& Couch e no crescimento de mudas de Eucalyptus. 1986. 51 f. Dissertação (Mestrado) Universidade Federal de Viçosa, Viçosa, 1986.

SOUZA, L.A. B. Eficiência de fungos ectomicorrízicos na absorção de fósforo e na promoção do crescimento de eucalipto. Pesquisa Agropecuária Brasileira, Brasília, v. 39, n. 4, p. 349-355, 2004.

SOUZA, L. A. B. et al. New isolates of ectomycorrhizal fungi and the growth of eucalypt. Pesquisa Agropecuária Brasileira, Brasília, v. 43, n. 2, p. 235-241, 2008.

SOUZA, L. A. B. Seleção de fungos ectomicorrízicos eficientes para promoção do crescimento de Eucalyptus dunnii Maiden. 2003. 117 f. Dissertação (Mestrado) - Universidade Federal de Santa Catarina, Florianópolis, 2003.

SUERTEGARAY, D. M. A.; GUASSELli, L. A.; VERDUM, R. Atlas da arenização: Sudoeste do Rio Grande do Sul. Porto Alegre: Secretaria da Coordenação e Planejamento, 2001. 85 p.

TEDESCO, M. J. et al. Análise de solo, plantas e outros materiais. 2. ed. Porto Alegre: UFRGS, 1995. $174 \mathrm{p}$.

TEIXEIRA, J. C. Modernização da agricultura no Brasil: impactos econômicos, sociais e ambientais. Revista Eletrônica da Associação dos Geógrafos Brasileiros, Três Lagoas, v. 2, n. 2, p. 21-42, 2005. 